\title{
villa en Ivrea
}

MARCELLO NIZZOLI y G. MARIO OLIVERI, arquitectos

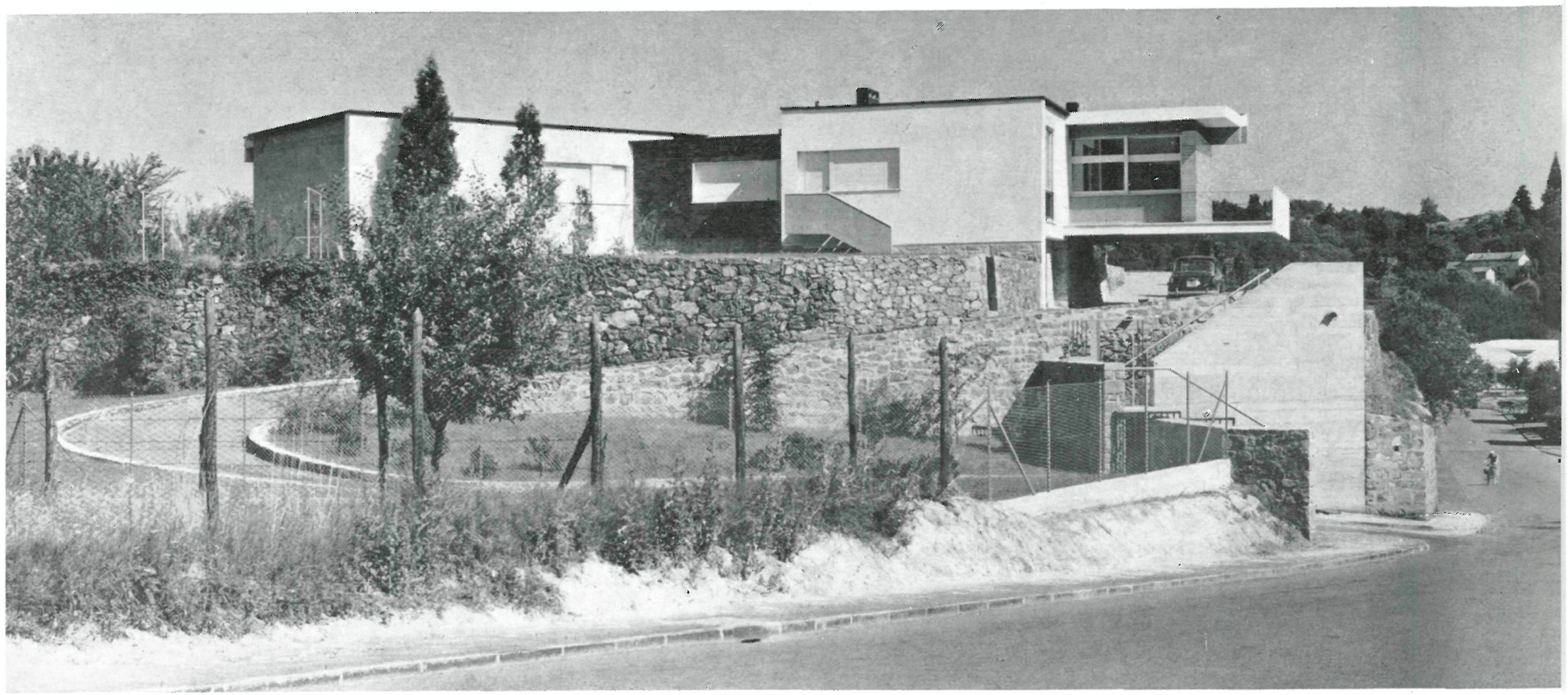

simopsis

$161 \cdot 105$

Se yergue sobre un terreno en pendiente. $\mathbf{Y}$, como es lógico y usual en estos casos, ha sido aprovechada esta circunstancia para crear una ambientación y movimiento interesantes. El total de edificación está dispuesto en dos plantas: Un semisótano, vestíbulo, estar-comedor, estudio, dormitorios, servicios y terrazas.

La estructura ha sido ejecutada a base de muros de mampostería careada con despiece irregular y machos resistentes de hormigón. Destacan, por su originalidad, las cubiertas planas volando en determinados puntos con formas sencillas y graciosas, que ayudan a romper la monotonía del conjunto.

En definitiva, la impresión que nos ofrece esta villa-de nítida blancura, sobre la que destacan las recortadas sombras producidas por los voladizos de cubierta-es sumamente agradable. La combinación de materiales-piedra, madera y paños de color-, junto a la movilidad de plantas y alzados, ofrecen un efecto plástico sorprendete y gracioso.

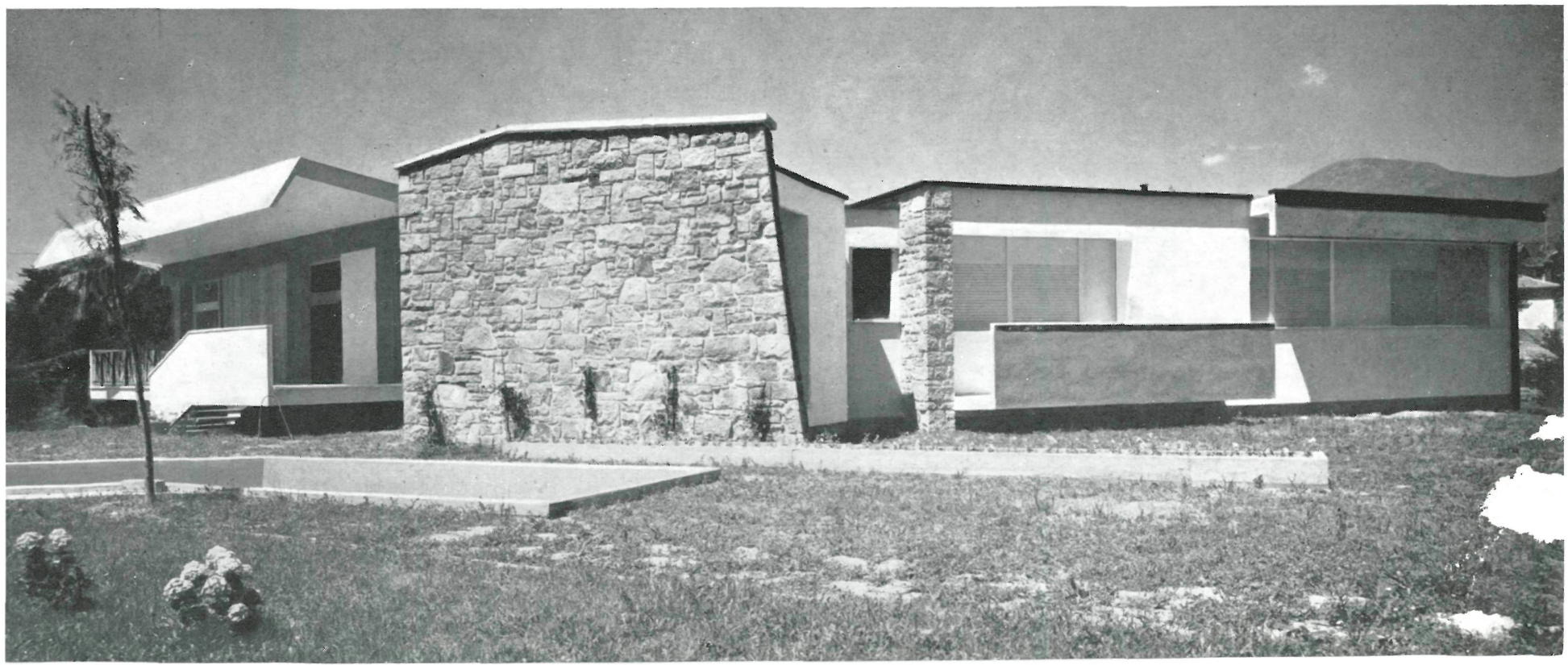




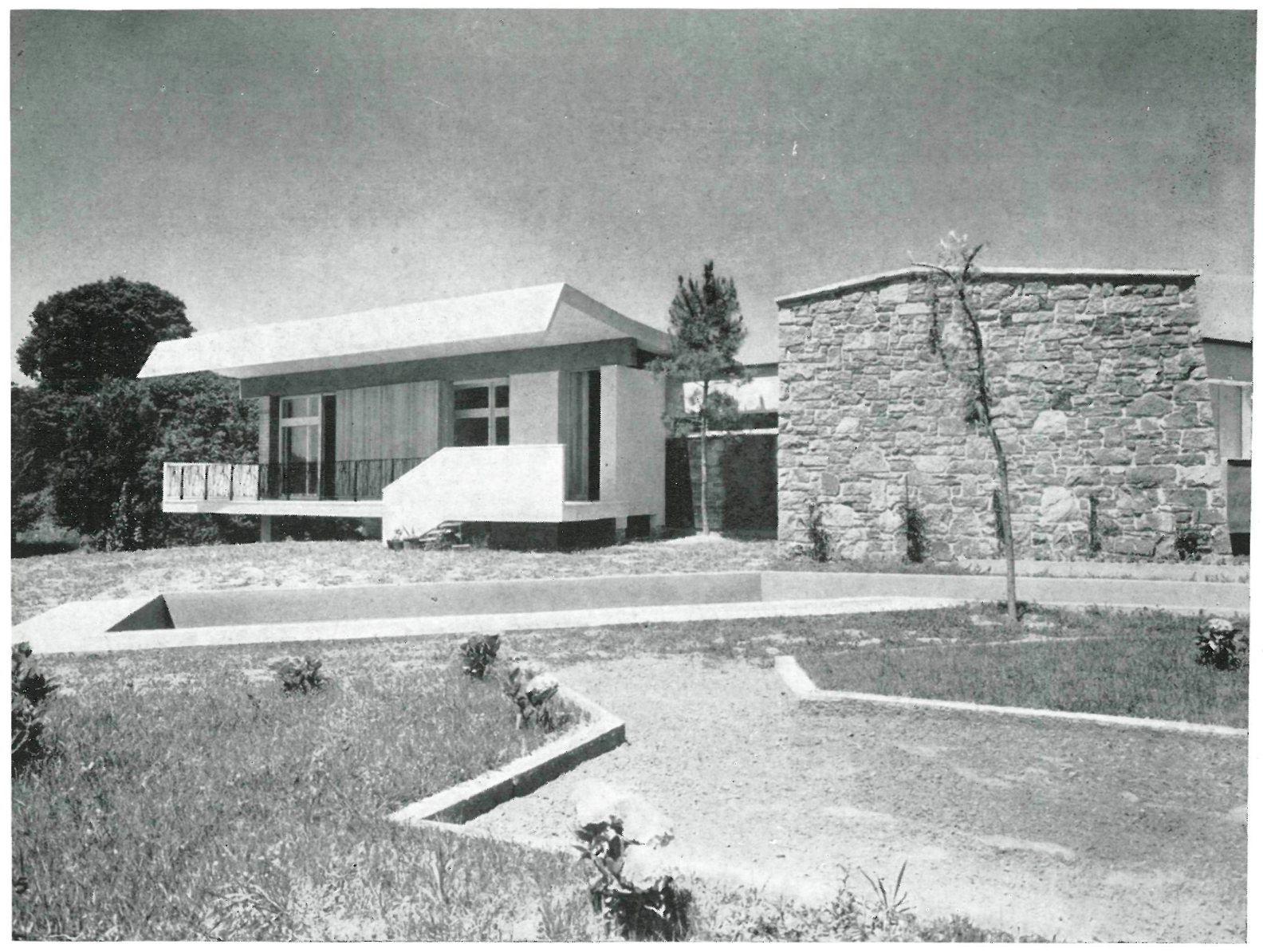

$\theta$

(4)

1

$\theta$

$-$

6

6

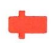

$x$

(4)
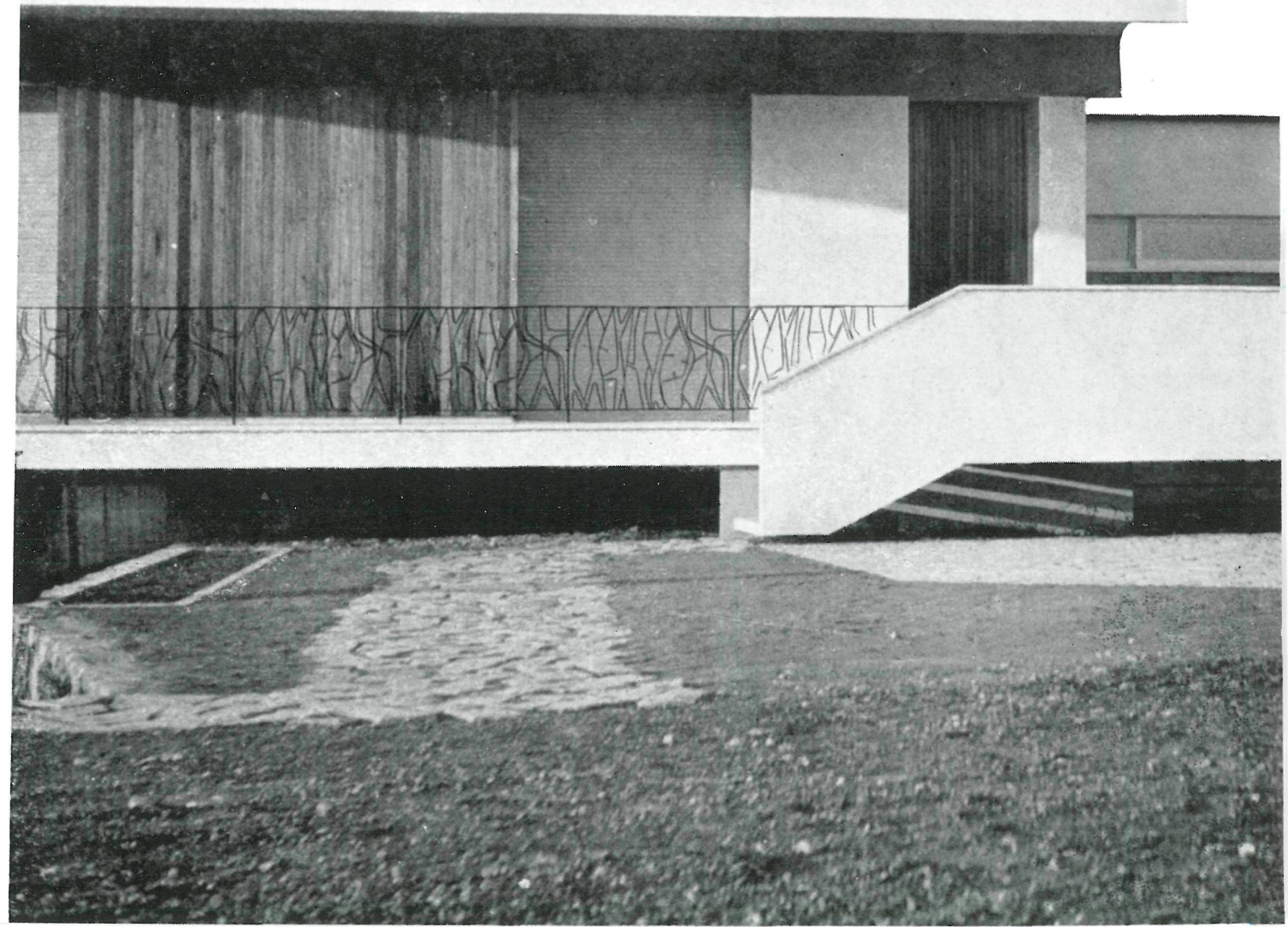
Ha sido edificada sobre un terreno, cuyo desnivel se aprovechó para lograr bellos contrastes $\mathrm{y}$ crear un paisaje interesante.

Consta de planta baja y semisótano sólo en una parte del mismo.

El semisótnno, cuya superficie útil es de $64,30 \mathrm{~m}^{2}$, tiene la forma de un rectángulo de $10,80 \times 8,00$. Aloja los cuartos de calefacción, una bodega de $4,80 \times 4,50 \mathrm{~m}$ y el garaje. Este último, con una escalera de acceso al piso superior.

La superficie útil de la planta baja es de $208,70 \mathrm{~m}^{2}$, que podemos dividir en tres zonas, dedicadas a estancia, servicios generales y dormitorios.

En la forma que adopta su planta, constituida por una serie de rectángulos organizados convenientemente, ha influido, considerablemente, el propósito de que las piezas principales gozasen de las orientaciones más adecuadas. Y, efectivamente, aparecen los dormitorios al E., la zona de estancia con orientación SE., quedando relegado el N. para las zonas de servicio.

El ala izquierda de la construcción está ocupada por una estancia, de $9,00 \times 4,30 \mathrm{~m}$, en la que, mediante la disposición del mobiliario y decoración, se ha logrado crear una serie de ambientes dentro de un mismo recinto. Unos sistemas de puertas correderas ponen en comunicación dicha estancia con el comedor, y con la amplia terraza en voladizo sobre la calzada para el tráfico interior de la villa.

$\mathrm{El}$ acceso al edificio se realiza por el bloque central, una distribución apropiada aisla la zona de servicio-cocina, dormitorio y aseo del servicio-del vestíbulo y resto de dependencias. Existe otro acceso desde el exterior, para el servicio, por la cocina.

El ala derecha está constituida por un dormitorio principal-con vestidor-, dormitorio de huéspedes-con terraza, baño, aseo-, y un estudio conectado con el vestíbulo.

La estructura ha sido ejecutada a base de muros de piedra y machones con pilastras de hormigón armado. 


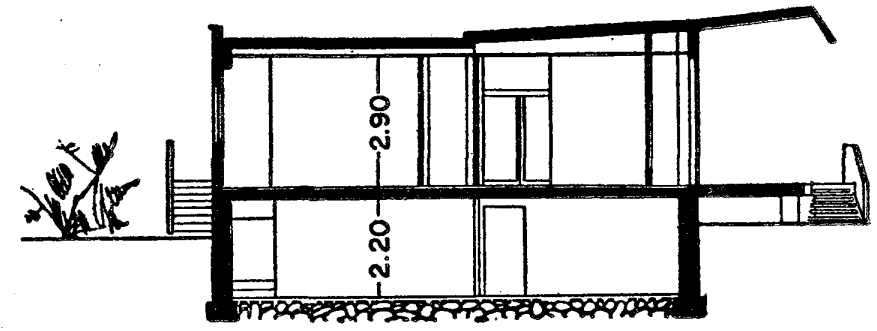

Ofrecen un interés especial las cubiertas planas, protegidas con una capa aislante impermeable. Aparecen a distintas alturas, formando una serie de planos que, en determinados puntos, vuelan con formas sencillas y graciosas, ayudando a romper la monotonía del conjunto.

Para los acabados exteriores han sido empleados: piedra, revoco y revestimientos de madera. La carpintería exterior es asimismo de madera y está provista de doble cristal y persianas arrollables. Merece mención la barandilla de hierro de la terraza-a la que se asoma la estanciaejecutada según un original diseño.

Cerca de la casa hay una amplia piscina de forma irregular, y el espacio restante-con excepción de las rampas y caminos de tráfico-está cubierto de césped entremezclado con las piedras, las cuales nos señalan sendas de circulación dentro del jardín.

Resumiendo, podemos decir: la impresión que nos ofrece esta villa--de blancura mediterránea sobre la que contrastan las recortadas sombras producidas por los voladizos de cubierta-es sumamente agradable.

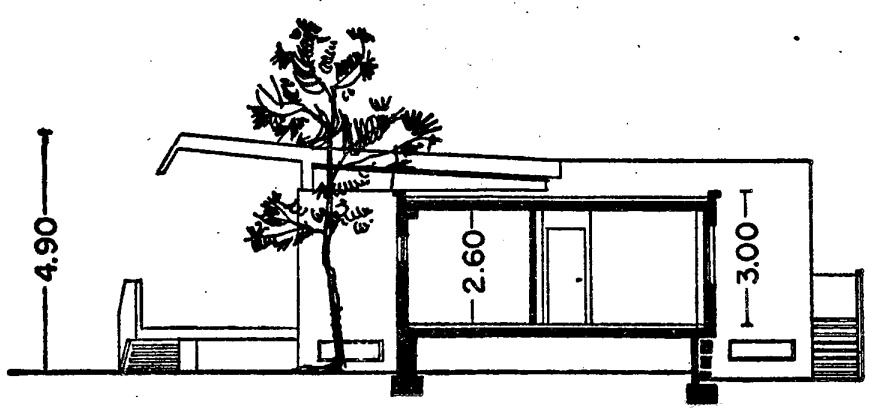



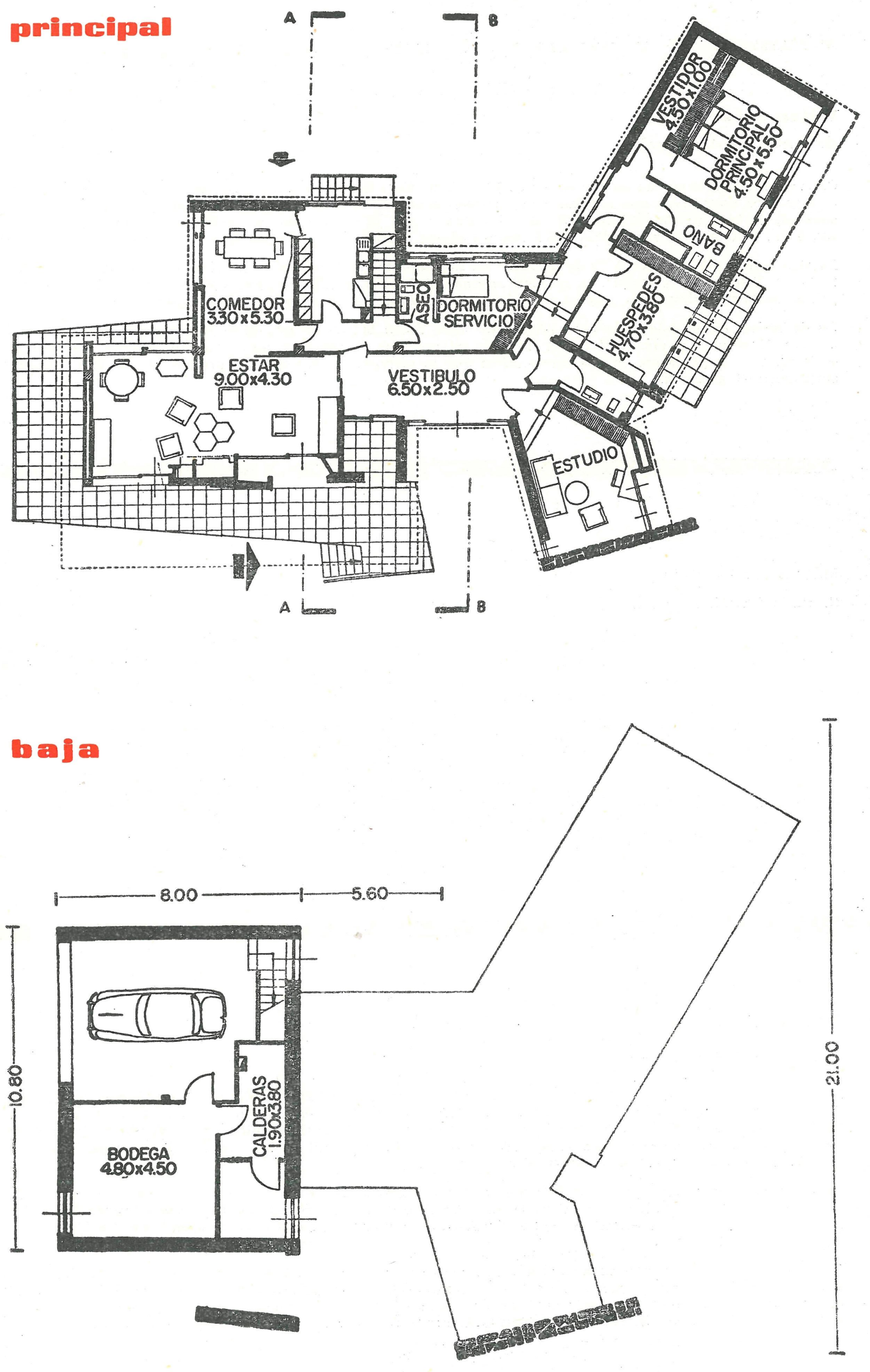


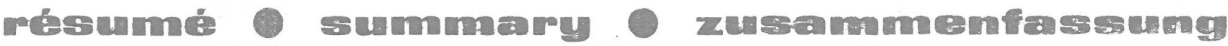

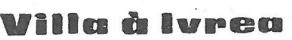

G. Mario Oliveri, architecte.

Cette villa est située sur un terrain en pente. Et, comme c'est logique et usuel dans ce cas, on a profité de cette circonstance pour créer une ambiance et un mouvement intéressants. L'édifice comprend deux étages: un sous-sol, de $64,30 \mathrm{~m}$ de surface utile, avec un garage, une cave, une salle de chauffage, et un étage noble, de $208,70 \mathrm{~m}$, distribué en vestibule, salle à manger-séjour, studio, chambres, services et terrasses.

La structure a été exécutée à l'aide de murs de maçonnerie de moellons bruts irréguliers et de piliers résistants de béton. On remarque, pour leur originalité, les couvertures pianes, en encorbellement en certains points, de formes simples et gracieuses, qui aident à rompre la monotonie de l'ensemble.

En définitive, l'impression que nous offre cette villa, d'une blancheur très pure sur laquelle se détachent les ombres découpés des encorbellements du toit, est agréable au plus haut point. La combinaison des matériaux - pierre, bois et panneaux en couleurs-, ainsi que la disposition des formes produisent un effet plastique surprenant et gracieux.

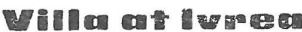

G. Mario Oliveri, architect.

This villa has been constructed on sloping ground, and naturally this circumstance has been utilised to give the design a dynamic and stimulating quality. There are two floor levels; a semibasement of $64.3 \mathrm{~ms}^{2}$ and the main part of the house, which has a surface of $208.7 \mathrm{~ms}^{2}$. The former includes a garage, celler and boiler room. The latter comprises the vestibule, a living cum dining room, study, bedrooms, services and terraces.

The construction consists mainiy of irregularly shaped, partly cut, stone walls, and load bearing concrete pillars. The roof is outstanding and original: it is a plane slab with overhangs in various directions, forming novel and intriguing configurations which heip to break any possible monotony of the building.

The overall impression conveyed by this villa, on whose pure white surfaces stand out the shaded projection of the roof outline, is most pleasant. The combination of materials, colour patterns, and the dynamic contrasts in shapes and volumes is surprising and charming.

\section{Vำ}

G. Mario Oliveri, Architekt.

Sie erhebt sich auf einem geneigten Gelände. Und da es in diesen Fällen logisch und herkömmlich ist, wurde diesar Umstand beniutzt, um eine interessante Umgebung und Bewegtheit zu schaffen. Das Gesamtgebäude besteht aus zwei Stockwerken: einem Fialbkeller von $64,30 \mathrm{~m}^{2}$ Nutzfläche mit Garage, Vorratskeller und Heizungsraum und einem vornehmen Geschoss von $208,70 \mathrm{~m}^{2}$, aufgeteilt in Vorraum, Wohn-Speisezimmer, Arbeitsraum, Schlafzimmer, Badezimmer und Terrassen.

Die Struktur wurde auf Grund von Mauerwerk aus Bruchsteinen mit unregelmässigen Stücken und widerständigen $\mathbb{K}$ ernen aus Beton ausgeführt. Es heben sich durch ihre Originalität die flachen Dächer hervor, die an gewissen Punkten mit einfachen und zierlichen Formen vorstehen und dazu beitragen, die Eintönigkeit des Ganzen zu unterbrechen.

Letzten Endes ist der Eindruck höchst angenehm, den uns diese Villa bietet, in ihrem reinen Weiss, worauf sich die von den Vorkragungen des Daches hervorgerufenen ausgeschnittenen Schatten abzeichnen. Die Materialzusammenstellung-Stein, Holz und färbige Wandscheiben-zusammen mit der Bewegtheit der Geschosse und Höhenaufrisse, rufen eine überraschende und zierliche plastische Wirkung hervor. 